\title{
Investigations on The Mechanism of Artificial Photosynthesis of Ca-Pc-PDI and Dendrimer Molecule by DFT Calculations
}

\author{
Rahmat Gunawan $^{1 *}$, Ulinnuha Hammamiyah ${ }^{1}$, Fahmi Fadillah ${ }^{1}$, \\ Chairul Saleh $^{2}$, and Saibun Sitorus ${ }^{2}$ \\ ${ }^{1}$ Physical Chemistry Laboratory, Dept. of Chemistry, Mulawarman University, \\ Samarinda 75123, Indonesia \\ ${ }^{2}$ Organic Chemistry Laboratory, Dept. of Chemistry, Mulawarman University, \\ Samarinda 75123, Indonesia \\ *E-mail: gunawan@fmipa.unmul.ac.id
}

\begin{abstract}
Artificial photosynthesis modelling of Ca-Pc-PDI complex Ca Phthalocyanine Perylenediimide), and dendrimer molecule using Density of Functional Theory (DFT) Method has been studied to showed the energy efficiency of these compounds in terms of electron transfer in photosynthesis. The Analysis of Ca-Pc-PDI and dendrimer compound and also chlorophyl has been done in all computations using the GAMESS-US software. The computations result in this research showed that the large wavelength complex compounds of Ca-Pc-PDI obtained was $138.3299 \mathrm{~nm}$ and energy efficiency obtained was $0.89 \mathrm{eV}$. The data analysis states that the absorption of harvest light energy of complex compounds Ca-Pc-PDI lies in the far UV spectrum. The other side, the polyphenylene dendrimer structure molecular orbital analyses it was found that the dendrimer was capable of electron transfers as indicated by the existence of HOMO and LUMO and result comparisons with chlorophyll. UV wavelengths of the polyethylene dendrimer and chlorophyll, respectively, suggesting that the polyphenylene dendrimer is capable of substituting chlorophyll in artificial photosyntheses. We can states from the result that these compound ability to be applied in the modeling of artificial photosynthesis as a material of energy absorption that mimics the workings of chlorophyll in terms of electron transfer in natural photosynthesis process.
\end{abstract}

Keywords: Artificial photosynthesis modelling, Ca-Phthalocyanine-Perylenediimide complex, and dendrimer molecule

\section{INTRODUCTION}

Energy is one of the most important factors in human life. The huge increase in fossil energy consumption that led to global warming and environmental pollution has been the primary concern of discussions on renewable energy resources and sustainable development (Vastra A, 2007). Various types of renewable energy research have been conducted and artificial photosynthesis is an example. Artificial photosynthesis is a chemical process that mimics the natural photosynthesis in which the conversion of sunlight, water, and carbon dioxide into carbohydrate and oxygen occurs.

The term photosynthesis is now adapted to refer to the capture and storage of energy from sunlight. In photosynthesis, chlorophyll is an absolutely vital part in absorbing light and transfering energy through energy resonance between chlorophyll pairs at the photosystem's reaction center (Qin Y, 2011). Artificial photosynthesis using visible light is one of the most wanted system for realization by scientists. During the last several decades, considerable efforts have gone into research in this relatively new field. Yet, continuous research remain necessary in order to find the ultimate method that provides the best energy efficiency possible (Haruo I., 2005).

Research in this field are still considered novelty and require significant funding in their development. Therefore, there is a need for more time-saving and cost-efficient alternatives in optimizing artificial photosynthesis efficiency along its development. One of the ways is by conducting research in silico, i.e. using mathematical calculations in computer programs to predict the atomic and molecular properties and the simulatory reactional pathways in either macroscopic or regular systems via computational chemistry experiments (Imahori, et al., 2004), (Bode BM \& Gordon MS, 1998). Photosynthetic processes involve electron transfers from a hydrolytic catalyst to an acceptor which are triggered by the sunlight-absorbing photosystem. This is the process which this 
research aimed to study through DFT methods on an artificial structure that mimicked the photosynthetic system commonly found in plants (Sastre SA, 2013).

\section{METHODS}

Electron Structure Calculation and Visualization The 3D molecular structure of Ca-Pc-PDI, the artificial photosynthetic compound, was built in Avogadro as shown by Figure (1). The structure was then optimized using B3LYP/6-31+G(d,p) methods through the combined works of Avogadro (Marcus et al., 2008) and GAMESS (Gordon MS \& Schimdt MW, 2005). An input file for electron structure computation with GAMESS was exported by Avogadro and the electron structure computation was run by GAMESS to find the optimum structure and its minimum energy.

The molecular orbital analysis was conducted with Gmolden (Dykstra, et al., 2008). From GAMESS calculation was visualized and the HOMO-LUMO energy data were analyzed. And the result from the previous step was visualized in WxMacMolPlt to check if the data obtained were convergent. If they are not, then an input file was rebuilt in WxMacMolPlt and re-run in GAMESS using the previously-obtained data. After convergence was reached, the result was then visualized in Gmolden to display the orbitals of the calculated structure (Schmidt MW \& Baldridge KK, 2009), (Young, 2008).

\section{RESULTS AND DISCUSSION}

\section{Structure initial of the molecule}

At this step, the 3D structure of Ca-Pc-PDI was optimized using steepest descent method which has the easiest and the most common algorithm. In addition, the force field parameter was also set to UFF (Universal Force Field). UFF was chosen since it took all types of atomic force fields found in the periodic table into account. This optimization process only fixed the atomic coordinates and the shape of Ca-Pc-PDI molecule while ignoring the energy calculation of each element and the interatomic bonding interactions. This process took about 90 minutes to reach the most stable energy of Ca-Pc-PDI in order to minimize the optimization workload of GAMESS with which the interatomic bonding energies were later calculated until convergence was reached.

Before optimization, the energy was
$529.172 \mathrm{~kJ} / \mathrm{mol}$ and after optimization with Avogadro it was significantly minimized to $3.994,5 \mathrm{~kJ} / \mathrm{mol}$. This showed that optimization had significant effect on the final result of the complex structure analysis for which the lower the energy, the more accurately the data were produced by the analysis, approaching an exact result.

\section{Structure optimization}

The energy-minimized structure was then optimized further using the more advanced DFT methods, and the basis set used was 6$31+\mathrm{G}(\mathrm{d}, \mathrm{p})$ due to its high enough precision.

The more precise a basis set is, the more accurate the calculation results will be. This basis set was also sufficient for geometry determination of such a large structure as $\mathrm{Ca}$ Pc-PDI complex. The SCFTYP parameter was set to UHF (Unrestricted Hatree-Fock). UHF is one of the most frequently-used calculation methods for analyzing molecular orbitals whose valence shell is not fully occupied nor giving up its electrons in a chemical bond during a reaction. Such molecular orbitals are called open shell orbitals in which electrons do not share the same spin value. UHF was preferred because it calculates the energy of each electron instead of electron pair as in RHF. Optimization tolerance is a gradient tolerance parameter that determines at what precision limit a convergent result shall be finalized. In this research, this structure optimization only took 6 days to complete. NSTEP indicates the maximum tolerable number of calculation steps taken to reach the optimum structure of a molecule, which in our case was Ca-Pc-PDI complex.

\section{Structure visualization}

\section{Ca-Pc-PDI complex}

After the complex was optimized and its energy was determined, the next step was to study the molecular orbitals of Ca-Pc-PDI complex. In this study, the first thing to do was to examine the electron transfer activity at the HOMO (highest occupied molecular orbital) and LUMO (lowest unoccupied molecular orbital) areas via Gmolden. Here are the visualizations of HOMO and LUMO areas in Ca-Pc-PDI complex, see Figure 1. 


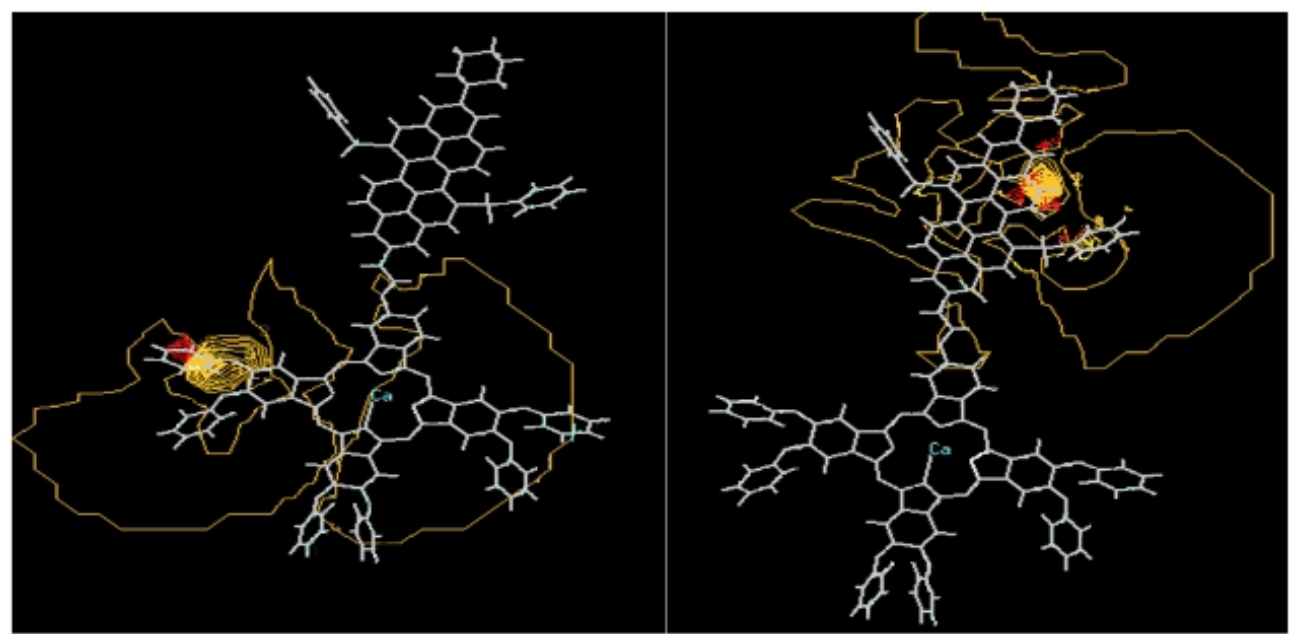

Figure 1. Visualization of HOMO energy level (Left) an LUMO energy level (Rigth) of Ca-PcPDI complex by GMolden.

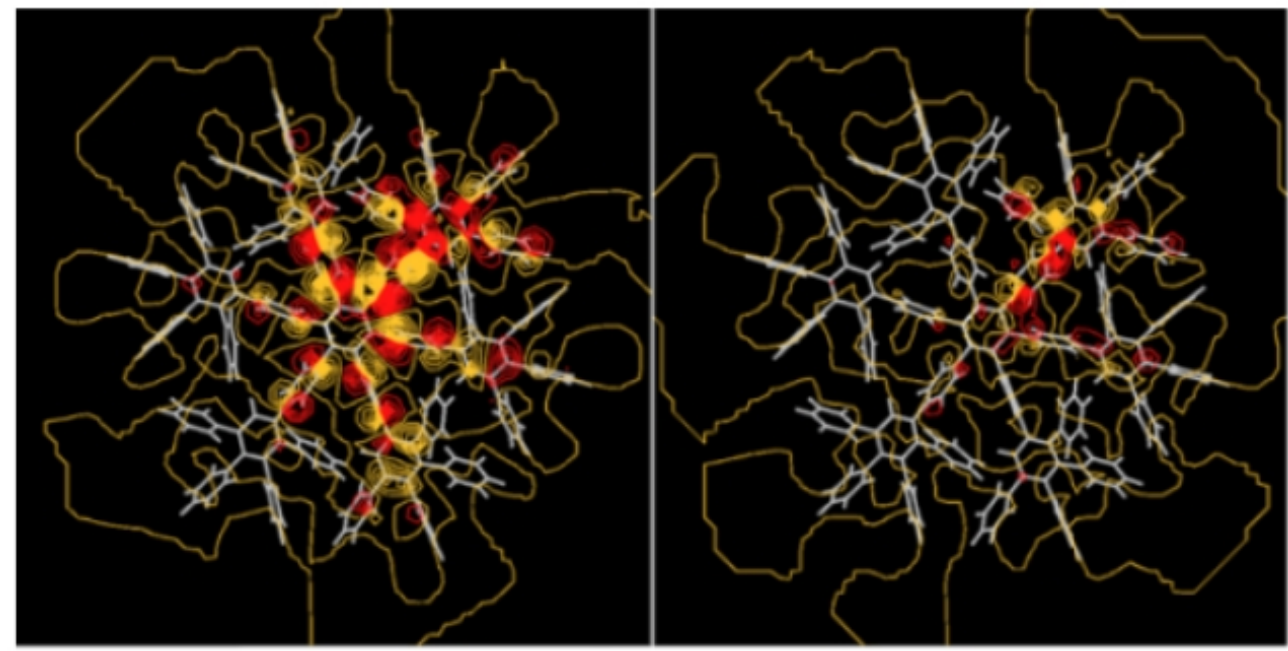

Figure 2. Visualization of HOMO energy level (Left) an LUMO energy level (Rigth) of Dendrimer molecule.

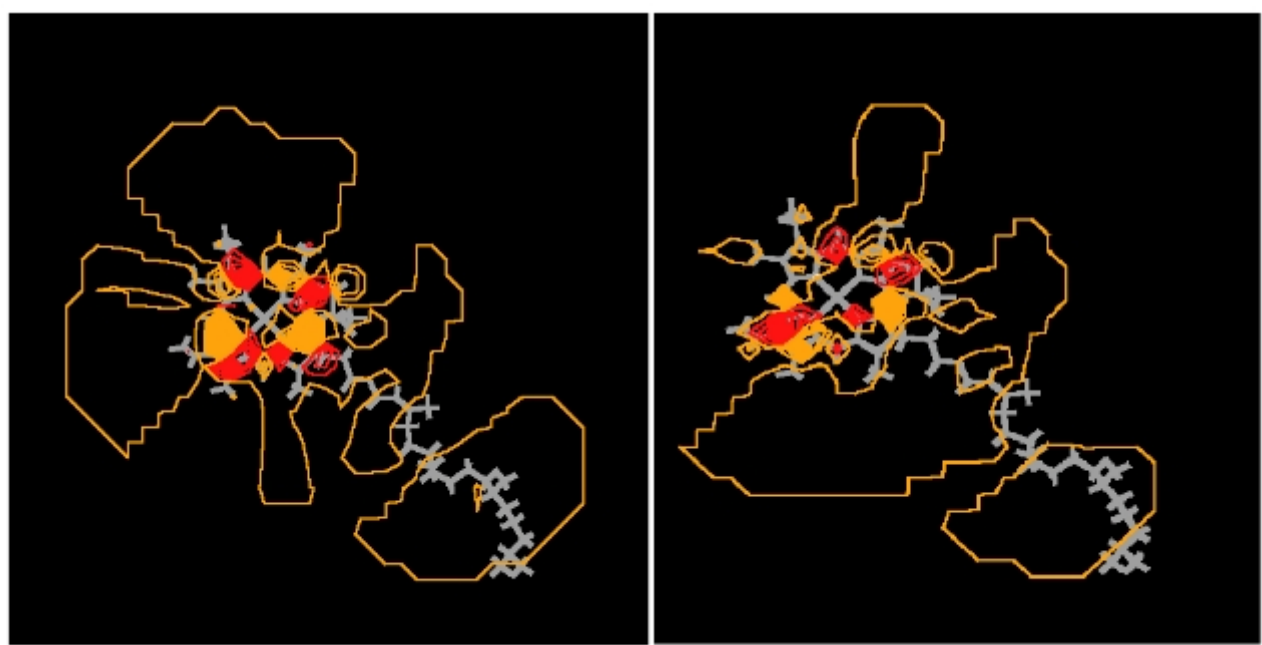

Figure 3. Visualization of HOMO energy level (Left) an LUMO energy level (Rigth) of chlorophyll molecule. 
In this case, electron transfer happened from HOMO area to its LUMO counterpart in Ca-Pc-PDI complex. The part of the molecule which had the highest occupation number (HOMO) would transfer electrons to the other part that had the lowest occupation number (LUMO). The locations of these parts are visible in the Gmolden visualization results for HOMO and LUMO areas as shown in Figure 1 (Left) and Figure 1 (Rigth), respectively. The visualizations show that there are HOMOLUMO areas in Ca-Pc-PDI complex. This indicates that there is electron activity in the complex. This further indicates that there happens a chemical reaction, a phenomenon in which a system consisting of atomic nuclei and electrons transforms in terms of either geometrical configuration or composition, including bonding and anti-bonding forces that are caused by electrons as well.

From the visualizations, it is seen that the HOMO area is located at the Caphthalocyanine molecule Figure 1 (Left) while the LUMO area at the perylendymide compound Figure 1 (Rigth). Electron transfers happen from HOMO to LUMO. The part of the complex that is in HOMO state, i.e. fully occupied by electrons, is disturbed by calcium which will then absorb energy from sunlight. This absorption will cause an excess of electrons to the HOMO area so that it has to donate some of its electrons to the acceptor part which is perylendymide. The distance between HOMO and LUMO areas was $20.813 \AA$, This indicates that such a long distance between HOMO and LUMO could raise a difficulty in electron transfer. However, in application, there will not only be a single molecule in use. There will be a lot of molecules instead. So, the possibility for electron transfers to happen from HOMO of a molecule to LUMO of another is still there. Therefore, this complex is still considerably effective for electron transfers.

Dendrimer molecule and chlorophyll

Structure optimization and molecular orbitals energy of both the polyphenylene dendrimer and chlorophyll with the help of Gmolden in revealing the sites where electron transfer activities might take place, i.e. the HOMOs and LUMOs of the compounds. Figure 2 and Figure 3, respectively visualize HOMO and LUMO of the polyphenylene dendrimer and chlorophyll. The HOMO of the polyphenylene dendrimer may transfer its electrons to the LUMO which is located in some separate spots within the molecule. In its most stable state, the polyphenylene dendrimer has a frequency of electron transfer activities similar to that of chlorophyll. This was indicated by the existence of HOMO and LUMO in the polyphenylene dendrimer molecule. Therefore, it can be assumed that the polyphenylene dendrimer may act as chlorophyll substitute in artificial photosyntheses. Figure 3 clearly shows that electron transfer activities may take place around the center of the cyclic part of chlorophyll and respectively visualize the HOMO and LUMO regions of a chlorophyll molecule.

The existence of HOMO and LUMO in chlorophyll molecules enables them to support electron transfer activities. Electron transfer is a key feature that singles out a potential chlorophyll substitute from the rest. This research has shown that the optimized structure of the polyphenylene dendrimer possessed HOMO and LUMO. This indicates that the compound may support electron transfers as well as chlorophyll does.

\section{Transfer electron analysis}

The HOMO-LUMO energy in Ca-Pc-PDI complex was found, from Gmolden visualization, to be $0,03296 \mathrm{eV}$ which represented the difference between HOMO and LUMO energies $\left(E_{\mathrm{LUMO}}-E_{\mathrm{HOMO}}\right)$. In order to get the wavelength, this value was converted to joule and the value of $0.1437 \times 10^{-}$ ${ }^{18} \mathrm{~J}$, was obtained. This was then substituted into Planck's equation, where $h$ is $\$ 6.626 \times 10^{-}$ $34 \mathrm{Js}^{-1}$ and $c$ is $3 \times 10^{-8} \mathrm{~ms}^{-1}$, to give a wavelength of $138.33 \mathrm{~nm}$, and see Figure 4 . 


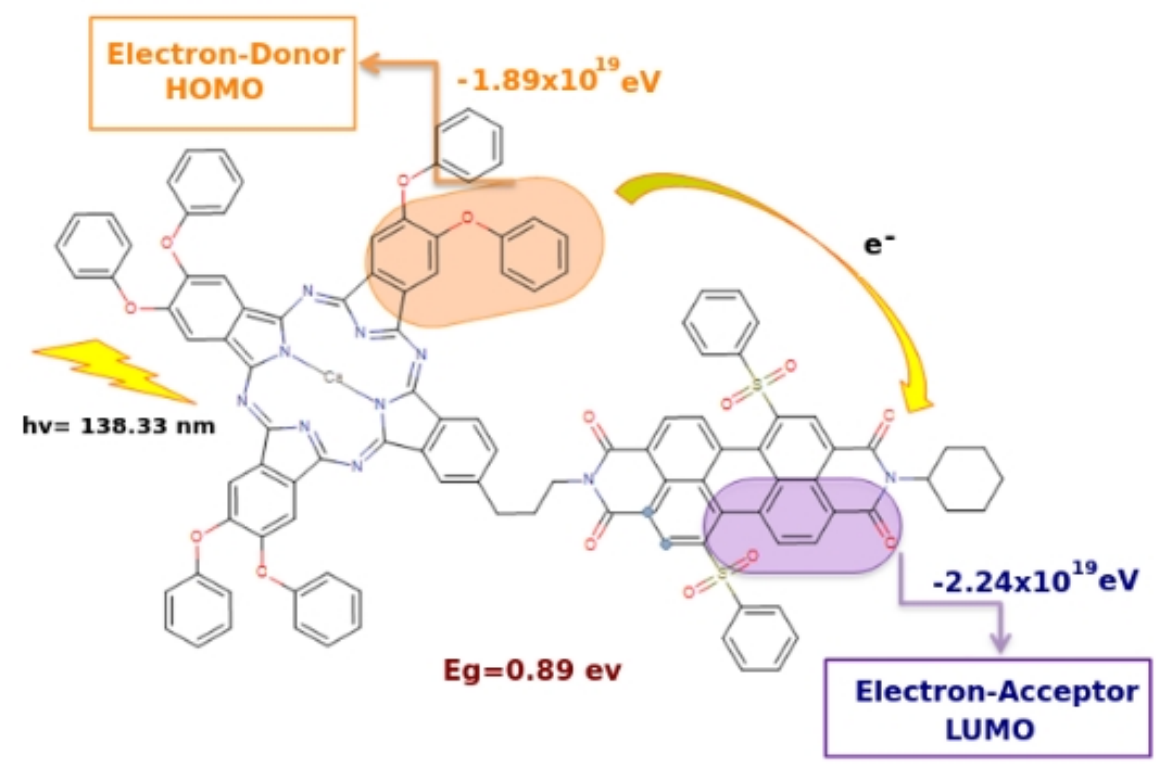

Figure 4. Energy gap result between HOMO and LUMO of Ca-Pc-PDI complex.

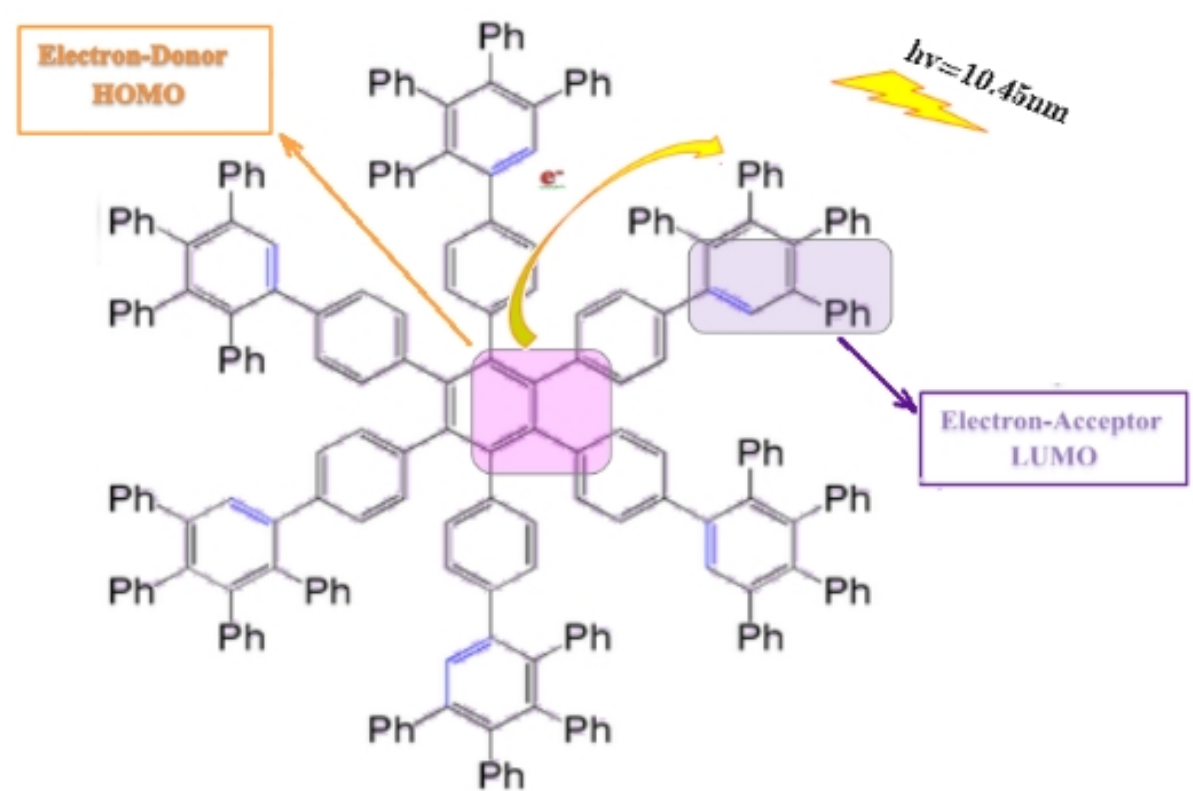

Figure 5. Energy gap result between HOMO and LUMO of Demdrimer molecule

Tabel 1. Comparation of Spectrum (HOMO to LUMO) between Ca-Pc-PDI complex and dendrimer molecule.

\begin{tabular}{|c|c|c|c|c|}
\hline Compounds & $\begin{array}{l}\Delta E \\
(\mathrm{~J})\end{array}$ & $\begin{array}{c}\text { Distance } \\
(\AA)\end{array}$ & $\begin{array}{c}\text { Emission } \\
(\mathrm{nm})\end{array}$ & Spectrum \\
\hline Chlorophyll & $1.18367 \times 10^{-18}$ & 5.67 & 36.7935 & UV \\
\hline $\begin{array}{l}\text { Ca-Pc-PDI } \\
\text { Complex }\end{array}$ & $0.1437 \times 10^{-18}$ & 20.813 & 138.3299 & UV \\
\hline $\begin{array}{l}\text { Dendrimer } \\
\text { molecule }\end{array}$ & $1.9022 \times 10^{-18}$ & 4.34 & 30.4500 & UV \\
\hline
\end{tabular}


Table 1 shows that the spectral range of both the polyphenylene dendrimer and chlorophyll falls within UV wavelength range. This means that both the polyphenylene dendrimer and chlorophyll are able to harvest UV wave, a type of electromagnetic wave in sunlight. Previous results also show that the polyphenylene dendrimer may undergo an intramolecular electron transfer. Therefore, it can be seen that the polyphenylene dendrimer meets a few criteria that qualify it as a chlorophyll substitute in artificial photosyntheses.

\section{CONCLUSION}

The energy absorption wavelength and energy efficiency of Ca-Pc-PDI complex were $138.3299 \mathrm{~nm}$ and $0,89 \mathrm{eV}$ respectively. Based on these data, it can be concluded that sunlight energy absorption by Ca-Pc-PDI complex shall occur within the far UV spectrum range. This compound is applicable to artificial photosynthesis modeling as an energyabsorbing material that mimicks the work of chlorophyll in terms of electron transfers. The result of this computational analysis approximates the exact value since it differs insignificantly from an experimental result value of $0.95 \mathrm{eV}$.

The polyphenylene dendrimer structure molecular orbital analyses it was found that the dendrimer was capable of electron transfers as indicated by the existence of HOMO and LUMO and result comparisons with chlorophyll. UV wavelengths of the polyethylene dendrimer and chlorophyll, respectively, suggesting that the polyphenylene dendrimer is capable of substituting chlorophyll in artificial photosyntheses.

\section{REFERENCES}

Thapper, S. Styring, G. Saracco, A. W. Rutherford, B. Robert, Ann Magnuson, W. Lubitz, A. Llobet, P. Kurz, A. Holzwarth, S. Fiechter, H. de Groot, S. Campagna, A. Braun, H. Bercegol, and Vincent Artero, 2013, Artificial Photosynthesis for Solar Fuels - an Evolving Research Field within AMPEA, a Joint Programme of the European Energy Research Alliance, Green., 3(1): 43-57

Brett M. Bodea, Mark S. Gordon,1998, Macmolplt: a graphical user interface for
GAMESS, Journal of Molecular Graphics and Modelling, 16(3): 133-138.

David Young, 2001, Computational Chemistry: A Practical Guide for Applying Techniques to Real World Problems, J. Am. Chem. Soc., 123(41): 10142-10143.

Dirk M. Guldi, Hiroshi Imahori, Koichi Tamaki, Yukiyasu Kashiwagi, Hiroko Yamada, Yoshiteru Sakata, and Shunichi Fukuzumi, 2004, A Molecular Tetrad Allowing Efficient Energy Storage for $1.6 \mathrm{~s}$ at $163 \mathrm{~K}, J$. Phys, Chem. A, 108(4): 541548.

Fukuzumi S, Ohkubo K, Ortiz J, Gutiérrez AM, Fernández-Lázaro F, Sastre-Santos A.,2008, Control of Photoinduced Electron Transfer in Zinc Phthalocyanine Perylenediimide Dyad and Triad by the Magnesium Ion, J Phys Chem A., 112(43): 10744-10752.

G. Schaftenaar, J.H. Noordik, 2000, Molden: A Pre- and Post-Processing Program for Molecular and Electronic Structures, Journal of Computer-Aided Molecular Design, 14(2): 123-134.

Haruo Inoue, Shigeaki Funyu, Yutaka Shimada, and Shinsuke Takagi, 2005, Artificial Photosynthesis Via Two-Electron Conversion: Photochemical Oxygenation Sensitized By Ruthenium Porphyrins With Water As Both Electron And Oxygen Atom Donor, J. Pure Appl, Chem. 774 (6): 10191033.

Marcus D Hanwell, Donald E Curtis, David C Lonie, Tim Vandermeersch, Eva Zurek and Geoffrey R Hutchison, 2012, Avogadro: an advanced semantic chemical editor, visualization, and analysis platform, Journal of Cheminformatics, 4(1): 1-17.

Michael W. Schmidt, Kim K. Baldridge, Jerry A. Boatz, Steven T. Elbert, Mark S. Gordon, Jan H. Jensen, Shiro Koseki, Nikita Matsunaga, Kiet A. Nguyen, Shujun $\mathrm{Su}$, Theresa L. Windus, Michel Dupuis and John A. Montgomery Jr., 1993, General atomic and molecular electronic structure system., Journal of Computational Chemistry, 14(11): 1347-1363.

Yuancheng Qin and Qiang Peng, 2012, Ruthenium Sensitizers and Their Applications in Dye-Sensitized Solar Cells, International Journal of Photoenergy, 201: 21-22. 